Göteborg ITP 95-23

September 1995

\title{
Gauged supersymmetric WZNW model using the BRST approach
}

\author{
Henric Rhedin円 \\ Institute of Theoretical Physics \\ Göteborg University and \\ Chalmers University of Technology \\ S-412 96 Göteborg, Sweden
}

\begin{abstract}
We consider the supersymmetric WZNW model gauged in a manifestly supersymmetric way. We find the BRST charge and the necessary condition for nilpotency. In the BRST framework the model proves to be a Lagrangian formulation of the supersymmetric coset construction, known as the $\mathrm{N}=1$ Kazama-Suzuki coset construction.
\end{abstract}

\footnotetext{
${ }^{1}$ hr@fy.chalmers.se
} 


\section{Introduction}

In the context of conformal field theory, much effort has been devoted to the WZNW model, especially gauged versions thereof, which are generally believed to describe all rational conformal field theories. In the gauged version the degrees of freedom of a subalgebra $h$ of the full algebra $g$ is removed by the procedure known as the gaugeing. On the algebra level, the counterpart is the coset conformal field theories known as the Goddard-Kent-Olive construction [1]. Those two approaches were connected when, in the BRST framework, the gauged WZNW model was shown to be the Lagrangian formulation of the algebraic approach [2, 3].

If we want to discuss realistic string theories, we would, however, like to incorporate fermions. This can be done by supersymmetrizing the WZNW model [4, 4 ]. The resulting action may be rewritten as a bosonic WZNW model and free fermions. In the algebraic approach, this may be formulated as a coset construction [6] in much the same way as for the bosonic case and we call this the Kazama-Suzuki coset construction. This construction gives an orthogonal decomposition of the $N=1$ superconformal algebra in the sense that it enables the definition of coset superconformal generators which decouple from the subalgebra generators. This was also discussed in [1] for the special case where the full diagonal algebra is taken as the subalgebra in the coset construction i.e. $(\mathrm{G} \otimes \mathrm{G}) / \mathrm{G}$ models. This provides with what is known as the minimal superconformal models.

Some time ago, a Lagrangian formulation of the minimal superconformal model was found by Schnitzer [7]. The starting point was the supersymmetric WZNW model. It was, however, gauged only by bosonic degrees of freedom and after the bosons and the fermions were decoupled. The resulting effective action is composed of the original bosonic WZNW model, an auxiliary bosonic WZNW model resulting from the gaugefields, the original fermions, and a fermionic ghost system. This resulted in a superconformal algebra which closed only in physical amplitudes. It was suggested that this lack of closure was due to the fact that the supersymmetric WZNW model had not been gauged in a manifestly supersymmetric way.

The manifestly supersymmetric gauging of the supersymmetric WZNW model was discussed in [8]. The effective action here consists of two supersymmetric WZNW models, the original as well as an auxiliary one originating from the gaugeing. Here it was also found that the conformal anomaly of the effective action of the gauged supersymmetric WZNW model coincided with that of the Kazama-Suzuki coset construction.

We here discuss the correspondence between the manifestly supersymmetrically 
gauged supersymmetric WZNW model and the Kazama-Suzuki coset construction. We approach this issue in the BRST framework. To this end, we introduce a superconformal ghost system which may be represented by a fermionic ghost system as well as a bosonic ghost system. The BRST symmetry of the effective action is found and the BRST charge is presented.

We proceed by showing that the generators of the $N=1$ superconformal algebra of both the full algebra and the subalgebra are BRST invariant. Furthermore, we find that the generators of the $N=1$ superconformal algebra of the effective action are given by the Kazama-Suzuli coset generators plus a BRST exact term. We hence provide with a Lagrangian formulation of the Kazama-Suzuki $N=1$ coset construction.

There exists, however, a different approach to the supersymmetric gauged WZNW model. One may decouple the bosons and the fermions and discuss the BRST symmetry of the resulting system. This gives us another model which resembles the one in []]. Specifically the two approaches yield different nilpotency conditions for the BRST charges and this results in different conformal anomalies. They, however, coincide for $\mathrm{g}=\mathrm{h}$. For $\mathrm{g} \neq \mathrm{h}$ the exact relation between those models is still unclear.

\section{Preliminaries}

We consider the supersymmetric WZNW model in superspace defined from the action 44, 5]

$$
S_{S W Z N W}=\frac{\hat{k}}{4 \pi} \int \mathrm{d} Z \mathrm{~d} \bar{Z}\left(-D \mathcal{G} \bar{D} \mathcal{G}^{-1}+\int \mathrm{d} t \mathcal{G} \frac{\mathrm{d}}{\mathrm{d} t} \mathcal{G}^{-1}\left(D \mathcal{G} \bar{D} \mathcal{G}^{-1}+\bar{D} \mathcal{G} D \mathcal{G}^{-1}\right)\right)
$$

in terms of the superfield $\mathcal{G}$ which may be expanded in components as $\mathcal{G}(Z, \bar{Z})=$ $g(z, \bar{z})+\theta \psi_{1}(z, \bar{z})+\bar{\theta} \psi_{2}(z, \bar{z})+\theta \bar{\theta} F(z, \bar{z})$. Here $g$ take values in a group $\mathrm{G}, \psi_{1}$ and $\psi_{2}$ are the components of a Majorana fermion, and $F$ is an auxiliary field. Also we have $D=\partial_{\theta}+\theta \partial_{z}, \bar{D}=\partial_{\bar{\theta}}+\bar{\theta} \partial_{\bar{z}}$. The equations of motion have the form $\bar{D}\left(D \mathcal{G} \mathcal{G}^{-1}\right)=0$. Written in components and using the equations of motions to eliminate the auxiliary field $F$ the action may be put in the form of an ordinary bosonic WZNW model defined over $g$ and free fermions $\lambda(z)=\psi_{1} g^{-1}$ and $\bar{\lambda}(\bar{z})=g^{-1} \psi_{2}$.

Superconformal transformations are generated by $K(Z)=\frac{1}{2} G(z)+\theta L(z)$ where $G(z)$ is the supersymmetry generator and $T(z)$ is the stress tensor obeying an $N=1$ superconformal algebra. 
The action is invariant under the superaffine transformations. The conserved current corresponding to this symmetry (we only bother to display the superholomorphic sector, the other sector is completely analogous) $j(Z)=-\frac{\hat{k}}{2} D \mathcal{G G}^{-1}$. In components we have $D \mathcal{G} \mathcal{G}^{-1}=\lambda+\theta\left(\partial_{z} g g^{-1}+\lambda \lambda\right) \equiv \lambda+\theta \hat{J}$ where we have used that $\bar{\lambda}$ only depends on $\bar{z}$. We have the superaffine operator product expansions (OPE's)

$$
\begin{aligned}
\hat{J}^{A}\left(z_{1}\right) \hat{J}^{B}\left(z_{2}\right) & =\frac{\hat{k} / 2}{\left(z_{1}-z_{2}\right)^{2}} \delta^{A B}+\frac{1}{z_{1}-z_{2}} i f_{C}^{A B} \hat{J}^{C}\left(z_{2}\right)+\text { r.t. } \\
\lambda^{A}\left(z_{1}\right) \lambda^{B}\left(z_{2}\right) & =\frac{\hat{k} / 2}{z_{1}-z_{2}} \delta^{A B}+\text { r.t. } \\
\hat{J}^{A}\left(z_{1}\right) \lambda^{B}\left(z_{2}\right) & =\frac{1}{z_{1}-z_{2}} i f_{C}^{A B} \lambda^{C}+\text { r.t.. }
\end{aligned}
$$

We have as usual taken $\hat{J}(z)=\hat{J}^{A}(z) t_{A}$ where $t_{A}$ are the generators of the Lie algebra $\mathrm{g}$ of $\mathrm{G}$ and $f^{A B}{ }_{C}$ are its structure constants.

It is convenient to decouple the fermions from the affine currents, and we hence introduce the new current $J^{A} \equiv \hat{J}^{A}+\frac{i}{\hat{k}} f^{A}{ }_{B C}: \lambda^{B} \lambda^{C}:$. (: : means as usual normal ordering.) $J$ obeys an affine OPE with level $\hat{k}-c_{g}$ and the OPE between $J$ and $\lambda$ consists of regular terms only. The superconformal generators may be realized in terms of $\lambda^{A}$ and $J^{A}$ as

$$
T(z)=\frac{1}{\hat{k}}: J_{A} J^{A}:-\frac{1}{\hat{k}}: \lambda_{A} \partial_{z} \lambda^{A}:, G(z)=\frac{2}{\hat{k}}: J^{A} \lambda_{A}:-\frac{2 i}{3 \hat{k}^{2}} f_{A B C}: \lambda^{A} \lambda^{B} \lambda^{C}: .
$$

In this form it is easy to check that $j^{A}(Z)$ is a superconformal weight one primary.

We now wish to consider the gauged supersymmetric WZNW model. We take it to be gauged by a subgroup $\mathrm{H}$, and in a manifestly supersymmetric way. The gauged action may be represented by two supersymmetric WZNW models, the original G valued supersymmetric WZNW model, and one valued over the subgroup $\mathrm{H}$ [8]. We will refer to the latter as the auxiliary supersymmetric WZNW model or shortly the auxiliary sector. We take the level of the auxiliary sector to be $\hat{\tilde{k}}$.

To be more precise we have in terms of the partitionfunction

$$
Z=\int[\mathrm{d} \mathcal{G}][\mathrm{d} \mathcal{A}][\mathrm{d} \overline{\mathcal{A}}] e^{S_{\hat{k}}(\mathcal{G}, \mathcal{A}, \overline{\mathcal{A}})}=\int[\mathrm{d} \mathcal{G}][\mathrm{d} \tilde{\mathcal{H}}] \mathcal{J} e^{S_{\hat{k}}(\mathcal{G})+S_{\hat{k}}(\tilde{\mathcal{H}})}
$$

where $\mathcal{A}$ and $\overline{\mathcal{A}}$ are the gauge superfields. $\mathcal{J}$ is the jacobian originating from a change of variables. It contributes to the conformal anomaly by a factor of $-3 d_{h}$ where $d_{h}$ is the dimension of the group H. Although the effective action was provided by Tseytlin the BRST analysis was not performed. 


\section{BRST analysis}

The jacobian $\mathcal{J}$ in (đ) may be represented by a ghost system consisting of fermionic ghosts $c, b$ of conformal weights $(0,1)$ and bosonic ghosts $\gamma, \beta$ of weights $\left(\frac{1}{2}, \frac{1}{2}\right)$. We take $c$ and $\gamma$ to have ghost number one and $b$ and $\beta$ to have minus one. Introducing the super ghostfields $C(Z)=c(z)+\theta \gamma(z)$ and $B(Z)=\beta(z)+\theta b(z)$ of weights $\left(0, \frac{1}{2}\right)$ and their antiholomorphic counterparts $\bar{C}(\bar{Z})=\bar{c}(\bar{z})+\bar{\theta} \bar{\gamma}(\bar{z})$ and $\bar{B}(\bar{Z})=\bar{\beta}(\bar{z})+\overline{\theta b}(\bar{z})$ we can replace the jacobian by a ghost action $S_{g h}=\int \mathrm{d}^{2} x \mathrm{~d}^{2} \theta(B \bar{D} C-\bar{B} D \bar{C})$.

In superfields the BRST transformation of the effective action is given by

$$
\begin{aligned}
& \delta_{B} \mathcal{G}=C \mathcal{G}-\mathcal{G} \bar{C}, \delta_{B} \widetilde{\mathcal{H}}=C \widetilde{\mathcal{H}}-\widetilde{\mathcal{H}} \bar{C}, \delta_{B} C=\frac{1}{2}\{C, C\}, \delta_{B} \bar{C}=\frac{1}{2}\{\bar{C}, \bar{C}\} \\
& \delta_{B} B=-\frac{\hat{k}}{2 \pi} D \mathcal{G} \mathcal{G}^{-1}-\frac{\hat{\tilde{k}}}{2 \pi} D \widetilde{\mathcal{H}} \widetilde{\mathcal{H}}^{-1}+[C, B], \delta_{B} \bar{B}=\frac{\hat{k}}{2 \pi} \mathcal{G}^{-1} D \mathcal{G}+\frac{\hat{\tilde{k}}}{2 \pi} \widetilde{\mathcal{H}}^{-1} D \widetilde{\mathcal{H}}+[\bar{C}, \bar{B}] .
\end{aligned}
$$

which is a straightforward generalization of Bastianelli's result for the bosonic case [9]. This transformation is trivially nilpotent for the $\mathcal{G}, C$, and $\bar{C}$ fields. For $B$ and $\bar{B}$ we have non-trivial transformations under $\delta_{B}^{2}$ e.g. we find $\delta_{B}^{2} \bar{B}=-\frac{\hat{k}}{2 \pi} \mathcal{G}^{-1} \bar{D} C \mathcal{G}-$ $\frac{\hat{\tilde{k}}}{2 \pi} \widetilde{\mathcal{H}}^{-1} \bar{D} C \tilde{\mathcal{H}}+\frac{\hat{k}+\hat{\tilde{k}}}{2 \pi} \bar{D} \bar{C}$. For nilpotency we thus need $\hat{k}+\hat{\tilde{k}}=0$ and make use of the holomorphisity of $C$. We get similar expressions for $\delta_{B}^{2} B$.

We may expand (5) in components to find the BRST transformation

$$
\begin{aligned}
& \delta_{B} g=c g-g \bar{c}, \delta_{B} h=c h-h \bar{c}, \delta_{B} c=\frac{1}{2}\{c, c\}, \delta_{B} \bar{c}=\frac{1}{2}\{\bar{c}, \bar{c}\} \\
& \delta_{B} b=\frac{\hat{k}}{2 \pi}\left(\partial_{z} g g^{-1}+\lambda \lambda\right)+\frac{\hat{\tilde{k}}}{2 \pi}\left(\partial_{z} h h^{-1}+\tilde{\lambda} \tilde{\lambda}\right)+[\beta, \gamma]+\{c, b\} \\
& \delta_{B} \bar{b}=\frac{\hat{k}}{2 \pi}\left(-g^{-1} \partial_{\bar{z}} g+\bar{\lambda} \bar{\lambda}\right)+\frac{\hat{\tilde{k}}}{2 \pi}\left(-h^{-1} \partial_{\bar{z}} h+\overline{\tilde{\lambda}} \overline{\tilde{\lambda}}\right)+[\bar{\beta}, \bar{\gamma}]+\{\bar{c}, \bar{b}\} \\
& \delta_{B} \lambda=-\gamma+\{c, \lambda\}, \delta_{B} \bar{\lambda}=\bar{\gamma}+\{\bar{c}, \bar{\lambda}\}, \delta_{B} \tilde{\lambda}=-\gamma+\{c, \tilde{\lambda}\}, \delta_{B} \overline{\tilde{\lambda}}=\bar{\gamma}+\{\bar{c}, \tilde{\tilde{\lambda}}\} \\
& \delta_{B} \gamma=[c, \gamma], \delta_{B} \bar{\gamma}=[\bar{c}, \bar{\gamma}], \delta_{B} \beta=-\frac{\hat{k}}{2 \pi} \lambda-\frac{\hat{\tilde{k}}}{2 \pi} \tilde{\lambda}+[c, \beta], \delta_{B} \bar{\beta}=\frac{\hat{k}}{2 \pi} \bar{\lambda}+\frac{\hat{\tilde{k}}}{2 \pi} \overline{\tilde{\lambda}}+[\bar{c}, \bar{\beta}]
\end{aligned}
$$

From this transformation we may find the BRST charge.

First we for convenience introduce the affine currents of the fermionic and the bosonic ghost systems $J_{F g h}^{a}(z) \equiv-i f_{c}^{a b}: c_{b} b^{c}:(z)$ and $J_{B g h}^{a}(z) \equiv i f_{c}^{a b}: \gamma_{b} \beta^{c}:(z)$. They will satisfy OPE's of affine type with levels $2 c_{h}$ and $-2 c_{h}$, respectively. The index convention is that capital indices are $\mathrm{g}$ valued and lower case $\mathrm{h}$ valued.

The BRST charge can now be written as

$$
\begin{aligned}
& Q \equiv \oint \frac{\mathrm{d} z}{2 \pi} j_{B R S T}(z)= \\
& \oint \frac{\mathrm{d} z}{2 \pi}:\left(c_{a}(z)\left(\hat{J}^{a}(z)+\hat{\tilde{J}} a(z)+\frac{1}{2} J_{F g h}^{a}(z)+J_{B g h}^{a}(z)\right):+\gamma_{a}(z)\left(\lambda^{a}(z)+\tilde{\lambda}^{a}(z)\right)\right) .
\end{aligned}
$$


If we demand that $Q$ is nilpotent we find the restriction $\hat{k}+\hat{\tilde{k}}=0$.

We may actually split the BRST charge (7) into two anticommuting and nilpotent pieces. Introduce the gradation $\operatorname{grad}\left(\gamma^{a}\right)=1, \operatorname{grad}\left(\beta^{a}\right)=-1$. This gives us $Q=Q_{0}+Q_{1}$ where $Q_{1}=\oint \frac{\mathrm{d} z}{2 \pi}\left(\gamma_{a}(z)\left(\lambda^{a}(z)+\tilde{\lambda}^{a}(z)\right)\right)$. This degree is conserved by the OPE or equivalently the commutator, hence $Q^{2}=0$ implies $Q_{0}^{2}=0, Q_{1}^{2}=0$ and $\left\{Q_{0}, Q_{1}\right\}=0$.

\section{Superconformal algebra}

The total superconformal generators of the effective action becomes

$$
\begin{aligned}
T^{t o t}(z)= & \frac{1}{\hat{k}}: \hat{J}^{A} \hat{J}_{A}:(z)-\frac{1}{\hat{k}}: \lambda^{A} \partial_{z} \lambda_{A}:(z)+\frac{1}{\hat{\tilde{k}}}: \hat{\tilde{J}}^{a} \hat{\tilde{J}}_{a}:(z)-\frac{1}{\hat{\tilde{k}}}: \tilde{\lambda}^{a} \partial_{z} \tilde{\lambda}_{a}:(z) \\
& -: b^{a} \partial_{z} c_{a}(z):+\frac{1}{2}: \partial_{z} \beta^{a} \gamma_{a}:(z)-\frac{1}{2}: \beta^{a} \partial_{z} \gamma_{a}:(z) \\
G^{t o t}(z)= & \frac{2}{\hat{k}}: \hat{J}^{A} \lambda_{A}:(z)-\frac{2 i}{3 \hat{k}^{2}} f_{A B C}: \lambda^{A} \lambda^{B} \lambda^{C}:(z)+ \\
& \frac{2}{\hat{\tilde{k}}}: \hat{\tilde{J}}^{A} \tilde{\lambda}_{A}:(z)-\frac{2 i}{3 \hat{\tilde{k}}^{2}} f_{A B C}: \tilde{\lambda}^{A} \tilde{\lambda}^{B} \tilde{\lambda}^{C}:(z)+\partial_{z} c_{a} \beta^{a}(z)-\gamma_{a} b^{a}(z) .
\end{aligned}
$$

The conformal anomaly of this system, using the nilpotency restriction $\hat{k}+\hat{\tilde{k}}=0$, reads $c_{t o t}=\frac{\left(\hat{k}-c_{g}\right) d_{g}}{\hat{k}}+\frac{1}{2} d_{g}-\frac{\left(\hat{k}-c_{h}\right) d_{h}}{\hat{k}}-\frac{1}{2} d_{h}$. This may be recognized as the conformal anomaly of the algebraic $\mathrm{N}=1$ coset construction of Kazama and Suzuki [6].

With the result of the gauged bosonic WZNW model at hand [2], this suggests that we should look for a decomposition of $T^{\text {tot }}(z)$ and $G^{\text {tot }}(z)$ such that $T^{\text {tot }}(z)=$ $T^{G / H}(z)+T^{e x}(z), G^{t o t}(z)=G^{G / H}(z)+G^{e x}(z)$ where $T^{G / H}(z) \equiv T^{G}(z)-T^{H}(z)$ and $G^{G / H}(z) \equiv G^{G}(z)-G^{H}(z)$ are the superconformal generators of the KazamaSuzuki coset construction. $T^{G}(z)$ and $G^{G}(z)$ are given in eq.(3), and $T^{H}(z)$ and $G^{H}(z)$ are provided below. Furthermore, we anticipate that $T^{e x}(z)$ and $G^{e x}(z)$ are BRST exact.

Following [6], we define $T^{H}(z)$ and $G^{H}(z)$ to be the generators which transforms the subalgebra pieces $\hat{J}^{a}(z)$ and $\lambda^{a}(z)$ as components of a weight one superfield. We must then define the new affine current $J^{\prime a}(z) \equiv \hat{J}^{a}(z)+\frac{i}{k} f_{b c}^{a}: \lambda^{b} \lambda^{c}:(z)$ which is of level $\hat{k}-c_{h}$.

We may now take the superconformal generators of the subalgebra to be given by (3) by interchangeing capital indices for lower case indices and changeing $J$ into $J^{\prime}$ [6]. This provides an orthogonal decomposition in the desired sense i.e. the OPE's $T^{G / H} \hat{J}^{a}, T^{G / H} \lambda^{a}, G^{G / H} \hat{J}^{a}, G^{G / H} \lambda^{a}$ all gives only regular terms. 
The BRST current $j_{B R S T}(z)$ transforms under the superconformal algebra as a conformal weight one field with a superpartner $u_{B R S T}(z)$ of conformal weight $1 / 2$. Explicitly we have $u_{B R S T}(z)=-c_{a}\left(\lambda^{a}+\tilde{\lambda}^{a}\right)(z)+\frac{i}{2} f_{c}^{a b}: c_{a} c_{b} \beta^{c}:(z)$. This means that we may construct the superconformal weight one field $u_{B R S T}(z)+\theta j_{B R S T}(z)$. The components satisfy OPE's which are trivial in the sense that, $j_{B R S T} u_{B R S T}$ gives only regular terms if and only if $\hat{k}+\hat{\tilde{k}}=0$ and $u_{B R S T} u_{B R S T}$ always gives only regular terms. Furthermore, we have that $u_{B R S T}(z)$ is BRST exact, indeed $u_{B R S T}(w)=\left[Q, c_{d} \beta^{d}(w)\right]$.

The BRST current obeys identical OPE's with the superconformal generators $T^{e x}$ and $G^{e x}$ as it does with $T^{t o t}$ and $G^{t o t}$. This means that $T^{t o t}, G^{t o t}, T^{e x}$ and $G^{e x}$ are all BRST invariant.

In order to find if $T^{e x}(z)$ is BRST exact it is convenient to work with the commutator $\{Q, K(z)\}$ of some operator $K(z)$. It is straightforward to verify that we indeed have $T^{e x}(z)=\left\{Q, K^{e x}(z)\right\}$, where $K^{e x}(z)$ is given by

$$
\begin{aligned}
& K^{e x}(z)= \\
& \frac{1}{\hat{k}} b_{a}\left(\hat{J}^{a}-\hat{\tilde{J}}^{a}\right)(z)+\frac{1}{2 \hat{k}}\left(\partial_{z} \beta_{a}\left(\lambda^{a}-\tilde{\lambda}^{a}\right)(z)-\beta_{a}\left(\partial_{z} \lambda^{a}-\partial_{z} \tilde{\lambda}^{a}\right)(z)\right)+ \\
& \frac{4 i}{3 \hat{k}^{2}} f_{a b c}\left(\beta^{a}\left(\lambda^{b}-\frac{1}{2} \tilde{\lambda}^{b}\right) \hat{J}^{c}(z)+\beta^{a}\left(\tilde{\lambda}^{b}-\frac{1}{2} \lambda^{b}\right) \hat{\tilde{J}}(z)+b^{a}\left(\frac{1}{2} \lambda^{b} \lambda^{c}+\frac{1}{2} \tilde{\lambda}^{b} \tilde{\lambda}^{c}-\frac{1}{2} \lambda^{b} \tilde{\lambda}^{c}\right)\right)
\end{aligned}
$$

The BRST exactness of the superpartner $G^{e x}(z)$ follows from the algebra and the graded Jacobi identity. Obviously $T^{e x}(z)$ and $G^{e x}(z)$ generates a superconformal algebra (with vanishing central charge) as may be seen from the fact that it corresponds to G/G gaugeing. Consider now the OPE $T^{e x}(z) G^{e x}(w)=\frac{3 / 2 G^{e x}(w)}{(z-w)^{2}}+$ $\frac{\partial_{w} G^{e x}(w)}{z-w}+$ r.t.. In terms of commutators this amounts to $\left[L_{m}^{e x}, G_{r}^{e x}\right]=\left(\frac{1}{2} m-r\right) G_{m+r}^{e x}$ where we have used the modes $L_{m}^{e x}$ and $G_{r}^{e x}$ of the Laurent expansion of $T^{e x}(z)$ and $G^{e x}(z)$ respectively. We have also that $L_{m}^{e x}=\left\{Q, K_{m}^{e x}\right\}$ in terms of the modes $K_{m}^{e x}$ of $K^{e x}(z)$. Putting this together, using the graded Jacobi identity and BRST invariance of $G^{e x}(z)$ we may find $\left(\frac{1}{2} m-r\right) G_{m+r}^{e x}=\left[L_{m}^{e x}, G_{r}^{e x}\right]=\left[\left\{Q, K_{m}^{e x}\right\}, G_{r}^{e x}\right]=$ $-\left[\left\{K_{m}^{e x}, G_{r}^{e x}\right\}, Q\right]$. Since $r$ and $m$ are both arbitrary it is clear that $G(z)$ is BRST exact.

\section{$5 \quad$ Remarks}

There exists a somewhat different approach to the BRST analysis of this model. We may, following Schnitzer [7], decouple the fermions in the sense that we in the pathintegral change variables from $\psi_{1}$ and $\psi_{2}$ to $\lambda$ and $\bar{\lambda}$. This gives rise to an anomaly 
which manifests itself as a shift in the level of the resulting bosonic WZNW model $\hat{k} \longrightarrow \hat{k}-c_{g}$. We hence end up with free fermions and the bosonic WZNW model with shifted level.

We use this procedure on the supersymmetric WZNW model gauged in the manifestly supersymmetric way [8]. What we find is two bosonic WZNW models, the original one of level $\hat{k}-c_{g}$ and the auxiliary of level $\hat{\tilde{k}}-c_{h}$. The free fermions remains as above and so does the ghost system.

The BRST charge decomposes into two mutually commuting pieces $Q=Q_{B}+Q_{\mathrm{ff}}$ corresponding to the bosonic WZNW models together with the fermionic ghosts, and the free fermion system together with the bosonic ghosts. The BRST symmetry corresonding to the first part is well known [2, 9]. We find a part of the BRST charge

$Q_{B}$ which squares to zero if $\hat{k}-c_{g}+\hat{\tilde{k}}-c_{h}+2 c_{h}=0$. The BRST charge of the free fermions $\lambda$ and $\tilde{\lambda}$ remains as above except that we introduce an scaling of $\tilde{\lambda}$ such that we have nilpotency for the level restriction $\hat{k}+\hat{\tilde{k}}=c_{g}-c_{h}$. This hence provides us with a different model than the one considered in the main part of this paper. They coincide, however, for $\mathrm{G}=\mathrm{H}$.

Acknowledgments We would like to thank Stephen Hwang for suggesting this project and for numerous discussions. Jens Lyng Petersen, Yu Ming and Jørgen Rasmussen of the Niels Bohr Institute are also acknowledged for discussions concerning this project. José Figueroa-O'Farrill and Sonia Stanciu of Queen Mary and Westfield College are acknowledged for discussions and for sharing their results on similar issues prior to publication.

\section{References}

[1] P. Goddard, A. Kent, and D. Olive, Virasoro algebras and coset space models, Phys. Lett. 152, 88; Unitary representations of the Virasoro and super-Virasoro algebras, Comm. Math. Phys. 103 (1986) 105

[2] D. Karabali, and H. Schnitzer, BRST quantization of the gauged WZW action and coset conformal field theories, Nucl. Phys. B329 (1990) 649

[3] S. Hwang and H. Rhedin, The BRST formulation of $G / H$ WZNW models, Nucl. Phys. B406 (1993) 165

[4] P. Di Vecchia, V.G. Knizhnik, J.L. Petersen and P. Rossi, A supersymmetric Wess-Zumino lagrangian in two dimensions, Nucl. Phys. B253 (1985) 701 
[5] E. Abdalla and M.C.B. Abdalla, Supersymmetric extension of the chiral model and the Wess-Zumino term in two dimensions, Phys. Lett. 152 (1985) 59

[6] Y. Kazama and H. Suzuki, New N=2 superconformal field theories and superstring compactification, Nucl. Phys. B321 (1989) 232;

Characterization of $N=2$ superconformal models generated by the coset space method, Phys. Lett. B216 (1989) 112

[7] H. Schnitzer, A path integral construction of superconformal field theories from a gauged supersymmetric Wess-Zumino-Witten action, Nucl. Phys. B324 (1989) 412

[8] A.A. Tseytlin, Conformal sigma models corresponding to gauged WessZumino-Witten theories, Nucl. Phys. B411 (1994) 509

[9] F. Bastianelli, BRST symmetry from a change of variables and the gauged WZNW model, Nucl. Phys. B361 (1991) 555 